\title{
Second-order total generalized variation based model for restoring images with mixed Poisson - Gaussian noise
}

\author{
Pham Cong Thanga, PhD, Lecturer, orcid.org/0000-0002-6428-102X, pcthang@dut.udn.vn \\ Tran Thi Thu Thao ${ }^{\mathrm{b}}$, M. Sc., Lecturer, orcid.org/0000-0001-7705-2405 \\ Nguyen Thanh Conga, M. Sc., Specialist, orcid.org/0000-0002-8060-0238 \\ Vo Duc Hoanga, PhD, Lecturer, orcid.org/0000-0002-6974-9023 \\ aThe University of Danang - University of Science and Technology, 54 Nguyen Luong Bang Street, \\ Danang 550000, Vietnam \\ bThe University of Danang - University of Economics, 71 Ngu Hanh Son Street, Danang 550000, Vietnam
}

\begin{abstract}
Introduction: A common problem in image restoration is image denoising. Among many noise models, the mixed Poisson Gaussian model has recently aroused considerable interest. Purpose: Development of a model for denoising images corrupted by mixed Poisson - Gaussian noise, along with an algorithm for solving the resulting minimization problem. Results: We proposed a new total variation model for restoring an image with mixed Poisson - Gaussian noise, based on second-order total generalized variation. In order to solve this problem, an efficient alternating minimization algorithm is used. To illustrate its comparison with related methods, experimental results are presented, demonstrating the high efficiency of the proposed approach. Practical relevance: The proposed model allows you to remove mixed Poisson - Gaussian noise in digital images, preserving the edges. The presented numerical results demonstrate the competitive features of the proposed model.
\end{abstract}

Keywords - image denoising, total variation, minimization, mixed Poisson - Gausian noise.

For citation: Pham C. T., Tran T. T. T., Nguyen T. C., Vo D. H. Second-order total generalized variation based model for restoring images with mixed Poisson - Gaussian noise. Informatsionno-upravliaiushchie sistemy [Information and Control Systems], 2021, no. 2, pp. 20-32. doi:10.31799/1684-8853-2021-2-20-32

\section{Introduction}

Image denoising is an important task in digital image processing. During the formation procedure, the image is usually degraded by noise. The denoising problem is to recover $u$ from an observed image $f$ with the size of $M \times N$. In literature, many types of noise generated by different devices and processes have been considered, e. g., Gaussian [1], Poisson [2], as well as mixed noise, e. g., mixed Poisson - Gaussian [3]. In practical, the Poisson - Gaussian model can accurately describe the noise present in a number of imaging applications such as astronomy, medicine, biology, etc... [4, 5]. The Poisson component accounts for the signal-dependent uncertainty inherent to the photon counting process, and the additive white Gaussian noise component accounts for the other signal-independent noise sources, such as thermal noise [6].

As is well known, several approaches have been developed for recovering images corrupted by the mixed Poisson - Gaussian noise. Among them, one of popular approaches is perhaps total variation (TV) model for mixed Poisson Gaussian noise removal (TVPG) [7, 8] using the TV norm as regularization term, formulated as follows:

$$
\begin{gathered}
u^{*}=\underset{u}{\arg \min }\left(\int_{\Omega}|\nabla u| \mathrm{d} x+\frac{\lambda}{2} \int_{\Omega}(u-f)^{2} \mathrm{~d} x+\right. \\
\left.+\beta \int_{\Omega}(u-f \log u) \mathrm{d} x\right),
\end{gathered}
$$

where $f$ is the observed image; $\Omega \subset \mathbb{R}^{2}$ be bounded open set and $u$ must be positive almost everywhere over $\Omega ; \lambda, \beta$ are positive regularization parameters.

In literature, we can find many efficient algorithms for solving the TV regularized mixed Poison - Gaussian denoising model (1), such as a primal-dual algorithm [9], an augmented Lagrangian method [10-12], the split Bregman method [13, 14], etc.

As is well known, the TV regularizer framework preserves edges well but has the transformation of smooth regions into piecewise constant regions. To avoid this problem, many regularization techniques for the denoising problem have been introduced, including non-local total variation [15], TV combined with higher-order term [16], Euler's elastic model [17], a mean curvature model [18, 19]. Reccently, a well-known method is the total generalized variation (TGV) introduced as penalty functional for image restoration [20, 21]. TGV includes higher-order derivatives of $u$. Image reconstructed by TGV regularization usually includes sharp edges and 
piecewise polynomial intensities [22]. With simplicity and prominence, the second-order TGV with weight $\alpha$ $\left(T G V_{\alpha}^{2}\right)$ based models have been widely researched recently, and achieved great successes in image processing [23-25]. Applied for image denoising, the resulting model is given by:

$$
u^{*}=\underset{u}{\operatorname{arcmin}}\left(T G V_{\alpha}^{2}(u)+\frac{\lambda_{1}}{2} \int_{\Omega}(u-f)^{2} \mathrm{~d} x\right)
$$

The model (2) was proposed in [23] for denoising image corrupted by Gaussian noise. Therefore, in case of mixed Poisson - Gaussian noise, the model itself cannot provide necessary accuracy for further data interpretation and analysis.

Inspired by the advantages of $T G V_{\alpha}^{2}$ regularization, we propose an second-order TGV regularized model for the mixed Poisson - Gaussian noise removal problem as follows:

In this paper, we employ the the second-order TGV instead of the standard TV norm in the model (1) and propose the following optimization problem:

$$
u^{*}=\underset{u}{\arg \min }\left(T G V_{\alpha}^{2}(u)+\frac{\lambda_{1}}{2} \int_{\Omega}(u-f)^{2} \mathrm{~d} x+\lambda_{2} \int_{\Omega}(u-f \log u) \mathrm{d} x\right),
$$

where $\lambda_{1}$ and $\lambda_{2}$ are positive parameter.

Our main contributions in this paper are following. We introduce a new total variation model for restoring image with mixed Poisson - Gaussian on the basis of the $T G V_{\alpha}^{2}$. The second important advantage is to extend an efficient alternating minimization method for solving the proposed model. Furthermore, we provide experimental results to demonstrate the high efficiency of our algorithm for considered problem, in comparison with related methods.

\section{Proposed method}

\section{The denoising model}

In this paper, we consider the following optimization problem (3):

$$
u^{*}=\underset{u}{\arg \min }\left(T G V_{\alpha}^{2}(u)+\frac{\lambda_{1}}{2} \int_{\Omega}(u-f)^{2} \mathrm{~d} x+\lambda_{2} \int_{\Omega}(u-f \log u) \mathrm{d} x\right) .
$$

Referring [20,24], we shortly review the concept of the second-order TGV. The definnitions can be found in Appendix.

Following the Refs. [7, 23-26], we have theorem (Theorem 1) for the considered model.

Theorem 1. The optimization problem (3) has a solution.

Proof: The proof will be given in the Appendix for completeness.

According to [20,23-25], the discrete $T G V_{\alpha}^{2}$ regularization of $u$ can be formulated as

$$
T G V_{\alpha}^{2}(u)=\underset{w}{\min } \alpha_{1}\|\nabla u-w\|_{1}+\alpha_{2}\|\varepsilon(w)\|_{1}
$$

where $w=\left(w_{1}, w_{2}\right)^{\mathrm{T}} ; \varepsilon(w)=(1 / 2)\left(\nabla w+\nabla w^{\mathrm{T}}\right)$.

The operators $\varepsilon(w)$ and $\nabla u$ can be expressed as follows:

$$
\nabla u=\left[\begin{array}{cc}
\nabla_{1} & u \\
\nabla_{2} & u
\end{array}\right] \text { and } \varepsilon(w)=\left[\begin{array}{cc}
\nabla_{1} w_{1} & \frac{1}{2}\left(\nabla_{2} w_{1}+\nabla_{1} w_{2}\right) \\
\frac{1}{2}\left(\nabla_{2} w_{1}+\nabla_{1} w_{2}\right) & \nabla_{2} w_{2}
\end{array}\right],
$$

where $\nabla=\left(\nabla_{1} ; \nabla_{2}\right), \nabla_{1}$ and $\nabla_{2}$ are derivative operators in the horizontal and vertical directions, respectively. 
According to the version of $T G V_{\alpha}^{2}$, the discrete version of the minimization problem (3) is given by

$$
u^{*}=\underset{u, w}{\arg \min }\left(\alpha_{1}\|\nabla u-w\|_{1}+\alpha_{2}\|\varepsilon(w)\|_{1}+\frac{\lambda}{2}\|u-f\|_{2}^{2}+\beta\langle 1, u-f \log u\rangle\right) .
$$

\section{Computational method}

In this section, we derive the numerical method for problem (4) in detail. By the classical augmented Lagrangian multiplier method [16, 17, 19-21], we introduce three new variables $(d, g, z)$ and rewrite the equation (4) in the constrained optimization problem as follows:

$$
\begin{gathered}
\min _{u, d, g, z}\left(\alpha_{1}\|d\|_{1}+\alpha_{2}\|g\|_{1}+\frac{\lambda}{2}\|z-f\|_{2}^{2}+\beta\langle 1, z-f \log z\rangle\right), \\
\text { s.t. } d=\nabla u-w, g=\varepsilon(w), z=u
\end{gathered}
$$

with

$$
d=\left[\begin{array}{l}
d_{1} \\
d_{2}
\end{array}\right] \text { and } g=\left[\begin{array}{ll}
g_{1} & g_{3} \\
g_{3} & g_{2}
\end{array}\right]
$$

The augmented Lagrangian functional for the constrained optimization problem (5) is defined as

$$
\begin{gathered}
\mathcal{L}\left(u, w, d, g, z, \theta_{1}, \mu_{2}, \xi_{3}\right)=\left(\alpha_{1}\|d\|_{1}+\alpha_{2}\|g\|_{1}+\frac{\lambda}{2}\|z-f\|_{2}^{2}+\beta\langle 1, z-f \log z\rangle-\langle\theta, d-\nabla u+w\rangle+\right. \\
\left.+\frac{\eta_{1}}{2}\|d-\nabla u+w\|_{2}^{2}-\langle\xi, g-\varepsilon(w)\rangle+\frac{\eta_{2}}{2}\|g-\varepsilon(w)\|_{2}^{2}-\langle\mu, z-u\rangle+\frac{\eta_{3}}{2}\|z-u\|_{2}^{2}\right)
\end{gathered}
$$

where $\eta_{1}, \eta_{2}, \eta_{3}$ - positive parameters; $\theta, \xi, \mu-$ with Lagrangian multipliers.

The discrete gradient $\nabla z$ and the second-order derivatives $\nabla^{2} u$ of an image $u$ for the pixel location ( $\left.i, j\right)$ in $u(i=1 . . . M ; j=1 . . . N)$ are defined like:

$$
\begin{gathered}
\nabla_{1} u_{i, j}=u_{i+1, j}-u_{i, j}, \nabla_{2} u_{i, j}=u_{i+1, j}-u_{i, j}, \\
\nabla_{1} u_{i, j}=\left(\nabla_{x} u_{i, j}, \nabla_{y} u_{i, j}\right),\left|\nabla u_{i, j}\right|=\sqrt{\left(\nabla_{1} u_{i, j}\right)^{2}+\left(\nabla_{2} u_{i, j}\right)^{2}} .
\end{gathered}
$$

The minimization method to solve the problem (6) can be expressed as follows:

$$
\left\{\begin{array}{l}
u^{(k+1)}=\underset{u}{\arg \min }\left(-\left\langle\theta^{(k)}, d^{(k)}-\nabla u+w^{(k)}\right\rangle+\frac{\eta_{1}}{2}\left\|d^{(k)}-\nabla u+w^{(k)}\right\|_{2}^{2}-\right. \\
\left.-\left\langle\mu^{(k)}, z^{(k)}-u\right\rangle+\frac{\eta_{3}}{2}\left\|z^{(k)}-u\right\|_{2}^{2}\right) ; \\
w^{(k+1)}=\underset{w}{\arg \min }\left(-\left\langle\theta, d^{(k)}-\nabla u^{(k+1)}+w\right\rangle+\frac{\eta_{1}}{2}\left\|d^{(k)}-\nabla u^{(k+1)}+w\right\|_{2}^{2}-\right. \\
\left.-\left\langle\xi, g^{(k)}-\varepsilon(w)\right\rangle+\frac{\eta_{2}}{2}\|g-\varepsilon(w)\|_{2}^{2}\right) ; \\
d^{(k+1)}=\underset{g}{\arg \min }\left(\alpha_{1}\|d\|_{1}-\left\langle\theta, d-\nabla u^{(k+1)}+w^{(k+1)}\right\rangle+\frac{\eta_{1}}{2}\left\|d-\nabla u^{(k+1)}+w^{(k+1)}\right\|_{2}^{2}\right) ; \\
g^{(k+1)}=\underset{z}{\arg \min }\left(\alpha_{2}\|g\|_{1}-\left\langle\xi^{(k)}, g-\varepsilon\left(w^{(k+1)}\right)\right\rangle+\frac{\eta_{2}}{2}\left\|g-\varepsilon\left(w^{(k+1)}\right)\right\|_{2}^{2}\right) ; \\
z^{(k+1)}=\underset{2}{\arg \min }\left(\frac{\lambda}{2}\|z-f\|_{2}^{2}+\beta\langle 1, z-f \log z\rangle-\left\langle\mu^{(k)}, z-u^{(k+1)}\right\rangle+\frac{\eta_{3}}{2}\left\|z-u^{(k+1)}\right\|_{2}^{2}\right)
\end{array}\right.
$$


with update for $\theta_{1}^{(k+1)}, \xi_{2}^{(k+1)}, \mu_{3}^{(k+1)}$ :

$$
\left\{\begin{array}{l}
\theta^{(k+1)}=\theta^{(k)}+\eta_{1}\left(\nabla u^{(k+1)}-d^{(k+1)}-w^{(k+1)}\right) \\
\xi^{(k+1)}=\xi^{(k)}+\eta_{2}\left(\varepsilon\left(w^{(k+1)}\right)-g^{(k+1)}\right) \\
\mu^{(k+1)}=\mu^{(k)}+\eta_{3}\left(u^{(k+1)}-z^{(k+1)}\right)
\end{array}\right.
$$

The $u$ subproblem in (7) is given by:

$$
\begin{aligned}
u^{(k+1)}= & \underset{u}{\arg \min }\left(-\left\langle\theta^{(k)}, d^{(k)}-\nabla u+w^{(k)}\right\rangle+\frac{\eta_{1}}{2}\left\|d^{(k)}-\nabla u+w^{(k)}\right\|_{2}^{2}-\left\langle\mu^{(k)}, z^{(k)}-u\right\rangle+\right. \\
& \left.+\frac{\eta_{3}}{2}\left\|z^{(k)}-u\right\|_{2}^{2}\right)=\frac{\eta_{1}}{2}\left\|d^{(k)}-\nabla u+w^{(k)}-\frac{\theta^{(k)}}{\eta_{1}}\right\|_{2}^{2}+\frac{\eta_{3}}{2}\left\|z^{(k)}-u-\frac{\mu^{(k)}}{\eta_{3}}\right\|_{2}^{2} .
\end{aligned}
$$

Thus, we get

$$
\eta_{1} \nabla^{\mathrm{T}}\left(\nabla u+\frac{\theta^{(k)}}{\eta_{1}}-d^{(k)}-w^{(k)}\right)+\eta_{3}\left(u+\frac{\mu^{(k)}}{\eta_{3}}-z^{(k)}\right)=0 .
$$

We can rewrite the equation (9) as follows:

$$
\left(\eta_{1} \nabla^{\mathrm{T}} \nabla+\eta_{3}\right) u^{(k+1)}=\eta_{1} \nabla^{\mathrm{T}}\left(d^{(k)}+w^{(k)}-\frac{\theta^{(k)}}{\eta_{1}}\right)+\eta_{3}\left(z^{(k)}-\frac{\mu^{(k)}}{\eta_{3}}\right) .
$$

It is obvious that system (10) is linear and symmetric positive definite, therefore $z^{(k+1)}$ can be efficiently solved by fast Fourier transform [18], under the periodic boundary conditions:

$$
u^{(k+1)}=F^{-1}\left(\frac{F\left(\eta_{1} \nabla^{\mathrm{T}}\left(d^{(k)}+w^{(k)}-\frac{\theta^{(k)}}{\eta_{1}}\right)+\eta_{3}\left(z^{(k)}-\frac{\mu^{(k)}}{\eta_{3}}\right)\right)}{\eta_{1} F\left(\nabla^{\mathrm{T}} \nabla\right)+\eta_{3}}\right),
$$

where $F$ and $F^{-1}$ are the forward and inverse Fourier transform operators.

The $w$ problem is

$$
\begin{gathered}
w^{(k+1)}=\underset{w}{\arg \min }\left(-\left\langle\theta^{(k)}, d^{(k)}-\nabla u^{(k+1)}+w\right\rangle+\right. \\
\left.+\frac{\eta_{1}}{2}\left\|d^{(k)}-\nabla u^{(k+1)}+w\right\|_{2}^{2}-\left\langle\xi^{(k)}, g^{(k)}-\varepsilon(w)\right\rangle+\frac{\eta_{2}}{2} \| g-\left.\varepsilon(w)\right|_{2} ^{2}\right)= \\
=\frac{\eta_{1}}{2}\left\|w+d^{(k)}-\nabla u^{(k+1)}-\left.\frac{\theta^{(k)}}{\eta_{1}}\right|_{2} ^{2}+\frac{\eta_{2}}{2}\right\| \varepsilon(w)-g^{(k)}+\left.\frac{\xi^{(k)}}{\eta_{2}}\right|_{2} ^{2} .
\end{gathered}
$$

Therefore, we get: 


$$
\left\{\begin{array}{l}
\eta_{1}\left(d_{1}^{(k)}-\nabla_{1} u^{(k+1)}+w_{1}-\frac{\theta_{1}^{(k)}}{\eta_{1}}\right)+\eta_{2} \nabla_{1}^{\mathrm{T}}\left(\nabla_{1} w_{1}-g_{1}+\frac{\xi_{1}^{(k)}}{\eta_{2}}\right)+ \\
+\eta_{2} \nabla_{2}^{\mathrm{T}}\left(\frac{1}{2}\left(\nabla_{2} w_{1}+\nabla_{1} w_{2}\right)-g_{3}+\frac{\xi_{3}^{(k)}}{\eta_{2}}\right)=0 ; \\
\eta_{1}\left(d_{2}^{(k)}-\nabla_{2} u^{(k+1)}+w_{2}-\frac{\theta_{2}^{(k)}}{\eta_{1}}\right)+\eta_{2} \nabla_{1}^{\mathrm{T}}\left(\frac{1}{2}\left(\nabla_{2} w_{1}+\nabla_{1} w_{2}\right)-g_{3}+\frac{\xi_{3}^{(k)}}{\eta_{2}}\right)+ \\
+\eta_{2} \nabla_{2}^{\mathrm{T}}\left(\nabla_{2} w_{2}-g_{2}+\frac{\xi_{2}^{(k)}}{\eta_{2}}\right)=0 .
\end{array}\right.
$$

We have:

$$
\left\{\begin{array}{l}
\left(\eta_{1} I+\eta_{2} \nabla_{1}^{\mathrm{T}} \nabla_{1}+\frac{\eta_{2}}{2} \nabla_{2}^{\mathrm{T}} \nabla_{2}\right) w_{1}+\frac{\eta_{2}}{2} \nabla_{2}^{\mathrm{T}} \nabla_{1} w_{2}=\eta_{1}\left(\nabla_{1} u^{(k+1)}-d_{1}^{(k)}+\frac{\theta_{1}^{(k)}}{\eta_{1}}\right)+ \\
+\eta_{2} \nabla_{1}^{\mathrm{T}}\left(g_{1}-\frac{\xi_{1}^{(k)}}{\eta_{1}}\right)+\eta_{2} \nabla_{2}^{\mathrm{T}}\left(g_{3}-\frac{\xi_{3}^{(k)}}{\eta_{1}}\right) ; \\
\frac{\eta_{2}}{2} \nabla_{1}^{\mathrm{T}} \nabla_{2} w_{1}+\left(\eta_{1} I+\frac{\eta_{2}}{2} \nabla_{1}^{\mathrm{T}} \nabla_{1}+\eta_{2} \nabla_{2}^{\mathrm{T}} \nabla_{2}\right) w_{2}=\eta_{1}\left(\nabla_{2} u^{(k+1)}-d_{2}^{(k)}+\frac{\theta_{2}^{(k)}}{\eta_{1}}\right)+ \\
+\eta_{2} \nabla_{1}^{\mathrm{T}}\left(g_{3}-\frac{\xi_{3}^{(k)}}{\eta_{2}}\right)+\eta_{2} \nabla_{2}^{\mathrm{T}}\left(g_{2}-\frac{\xi_{2}^{(k)}}{\eta_{2}}\right) .
\end{array}\right.
$$

From (13), we have a system of linear equations in two unknowns $w_{1}^{(k+1)}, w_{2}^{(k+1)}$ :

$$
\left[\begin{array}{ll}
a & b \\
c & d
\end{array}\right]\left[\begin{array}{l}
w_{1}^{(k+1)} \\
w_{2}^{(k+1)}
\end{array}\right]=\left[\begin{array}{l}
s \\
t
\end{array}\right]
$$

with

$$
\begin{gathered}
a=\left(\eta_{1} I+\eta_{2} \nabla_{1}^{\mathrm{T}} \nabla_{1}+\frac{\eta_{2}}{2} \nabla_{2}^{\mathrm{T}} \nabla_{2}\right) ; \quad b=\frac{\eta_{2}}{2} \nabla_{2}^{\mathrm{T}} \nabla_{1} ; c=\frac{\eta_{2}}{2} \nabla_{1}^{\mathrm{T}} \nabla_{2} ; \\
d=\left(\eta_{1} I+\frac{\eta_{2}}{2} \nabla_{1}^{\mathrm{T}} \nabla_{1}+\eta_{2} \nabla_{2}^{\mathrm{T}} \nabla_{2}\right) ; \\
s=\eta_{1}\left(\nabla_{1} u^{(k+1)}-d_{1}^{(k)}+\frac{\theta_{1}^{(k)}}{\eta_{1}}\right)+\eta_{2} \nabla_{1}^{\mathrm{T}}\left(g_{1}-\frac{\xi_{1}^{(k)}}{\eta_{1}}\right)+\eta_{2} \nabla_{2}^{\mathrm{T}}\left(g_{3}-\frac{\xi_{3}^{(k)}}{\eta_{1}}\right), \\
t=\eta_{1}\left(\nabla_{2} u^{(k+1)}-d_{2}^{(k)}+\frac{\theta_{2}^{(k)}}{\eta_{1}}\right)+\eta_{2} \nabla_{1}^{\mathrm{T}}\left(g_{3}-\frac{\xi_{3}^{(k)}}{\eta_{2}}\right)+\eta_{2} \nabla_{2}^{\mathrm{T}}\left(g_{2}-\frac{\xi_{2}^{(k)}}{\eta_{2}}\right) .
\end{gathered}
$$

Similar to the $u$ subproblem, we can solve problems (14) with fast Fourier transform, under the periodic boundary conditions:

$$
w_{1}^{(k+1)}=F^{-1}\left(\frac{F(s d-b t)}{F(a d-c b)}\right) ; w_{2}^{(k+1)}=F^{-1}\left(\frac{F(a t-c s)}{F(a d-c b)}\right)
$$


The $d$ subproblem is given by:

$$
\begin{gathered}
d^{(k+1)}=\underset{d}{\arg \min }\left(\alpha_{1}\|d\|_{1}-\left\langle\theta, d-\nabla u^{(k+1)}+w^{(k+1)}\right\rangle+\frac{\eta_{1}}{2}\left\|d-\nabla u^{(k+1)}+w^{(k+1)}\right\|_{2}^{2}\right)= \\
=\underset{d}{\arg \min }\left(\alpha_{1}\|d\|_{1}+\frac{\eta_{1}}{2}\left\|d-\nabla u^{(k+1)}+w^{(k+1)}-\frac{\theta^{(k)}}{\eta_{1}}\right\|_{2}^{2}\right) .
\end{gathered}
$$

The solution of the $d$ subproblem can readily be obtained by applying the soft thresholding operator [27]:

$$
d^{(k+1)}=\frac{\nabla u^{(k+1)}-w^{(k+1)}+\frac{\theta^{(k)}}{\eta_{1}}}{\left|\nabla u^{(k+1)}-w^{(k+1)}+\frac{\theta^{(k)}}{\eta_{1}}\right|} \cdot \max \left(\left|\nabla u^{(k+1)}-w^{(k+1)}+\frac{\theta^{(k)}}{\eta_{1}}\right|-\frac{\alpha_{1}}{\eta_{1}}, 0\right)
$$

The g subproblem is given by:

$$
\begin{gathered}
g^{(k+1)}=\underset{g}{\arg \min }\left(\alpha_{2}\|g\|_{1}-\left\langle\xi^{(k)}, g-\varepsilon\left(w^{(k+1)}\right)\right\rangle+\frac{\eta_{2}}{2}\left\|g-\varepsilon\left(w^{(k+1)}\right)\right\|_{2}^{2}\right)= \\
=\underset{g}{\arg \min }\left(\alpha_{2}\|g\|_{1}+\frac{\eta_{2}}{2}\left\|g-\varepsilon\left(w^{(k+1)}\right)-\frac{\xi^{(k)}}{\eta_{2}}\right\|_{2}^{2}\right)
\end{gathered}
$$

The solution of the $g$ subproblem can be obtained by applying the soft thresholding operator too:

$$
g^{(k+1)}=\frac{\varepsilon\left(w^{(k+1)}\right)+\frac{\xi^{(k)}}{\eta_{2}}}{\left|\varepsilon\left(w^{(k+1)}\right)+\frac{\xi^{(k)}}{\eta_{2}}\right|} \cdot \max \left(\left|\varepsilon\left(w^{(k+1)}\right)+\frac{\xi^{(k)}}{\eta_{2}}\right|-\frac{\alpha_{2}}{\eta_{2}}, 0\right)
$$

The $z$ subproblem is given by:

$$
\begin{gathered}
z^{(k+1)}=\underset{z}{\arg \min }\left(\frac{\lambda}{2}\|z-f\|^{2}+\beta\langle 1, z-f \log z\rangle-\left\langle\rho_{3}^{(k)}, z-u^{(k+1)}\right\rangle+\frac{\eta_{3}}{2}\left\|z-u^{(k+1)}\right\|_{2}^{2}\right)= \\
\quad \underset{z}{\arg \min }\left(\frac{\lambda}{2}\|z-f\|_{2}^{2}+\beta\langle 1, z-f \log z\rangle+\frac{\eta_{3}}{2}\left\|z-u^{(k+1)}-\frac{\rho_{3}^{(k)}}{\eta_{3}}\right\|_{2}^{2}\right) .
\end{gathered}
$$

Therefore, we get

$$
\lambda(z-f)+\beta\left(1-\frac{f}{z}\right)+\eta_{3}\left(z-u^{(k+1)}\right)-\rho_{3}^{(k)}=0
$$

This equation can be rewritten as follows:

$$
\left(\lambda+\eta_{3}\right) z^{2}-z\left(\eta_{3} u^{(k+1)}+\rho_{3}^{(k)}-\beta+\lambda f\right)-\beta f=0
$$


The solution of $z^{(k+1)}$ is the positive solution given by:

$$
z^{(k+1)}=\frac{\left(\eta_{3} u^{(k+1)}+\rho_{3}^{(k)}-\beta+\lambda f\right)+\sqrt{\left(\eta_{3} u^{(k+1)}+\rho_{3}^{(k)}-\beta+\lambda f\right)^{2}+4\left(\eta_{3}+\lambda\right) \beta f}}{2\left(\eta_{3}+\lambda\right)} .
$$

The complete method is summarized in Algorithm 1. We need a stopping criterion for the iteration: we end the loop if the maximum number of allowed outer iterations $N$ has been carried out (to guarantee an upper bound on running time) or the following condition is satisfied for some prescribed tolerance $\sigma$ :

$$
\frac{\left\|u^{(k)}-u^{(k-1)}\right\|_{2}}{\left\|u^{(k)}\right\|_{2}}<\varsigma
$$

where $\sigma$ is a small positive parameter.

Algorithm 1: Alternating minimization method for solving the model (5).

1. Initialize: $z^{(0)}=u^{(0)}=f ; d^{(0)}=g^{(0)}=0 ; w^{(0)}=0 ; k=0$.

2. While Stopping condition is not satisfied do:

3 . Compute $u^{(k+1)}$ according to (11).

4. Compute $w^{(k+1)}$ according to (15).

4. Compute $d^{(k+1)}$ according to (16).

5 . Compute $g^{(k+1)}$ according to (17).

6 . Compute $z^{(k+1)}$ according to (18).

7. Update $\theta_{1}^{(k+1)}, \mu_{2}^{(k+1)}, \xi_{3}^{(k+1)}$ by (8).

10. $k=k+1$.

11. Endwhile.

12. Return $u$.

\section{Numerical experiments}

In this section, we present some numerical results to illustrate the performance of the proposed model for MPGN removal. In order to prove the superiority of the proposed model, we compare our results with closely related approaches [8, 23]: the TVPG model (1) and TGV model (2). For compared models, the optimization problem are implemented by the state-of-the-art alternating minimization algorithm. The original test images are shown in Fig. $1, a-d$.

All experiments were carried out in Windows 10 and Matlab running on a desktop equipped with an Intel Corei3, $2.1 \mathrm{GHz}$ and $12 \mathrm{~GB}$ of RAM. To assess quality of the restoration results, we use peak signal-to-noise ratio (PSNR) defined as follows:

$$
P S N R=10 \log _{10}\left(\frac{255^{2} \cdot M N}{\left\|u^{*}-u\right\|_{2}^{2}}\right)
$$
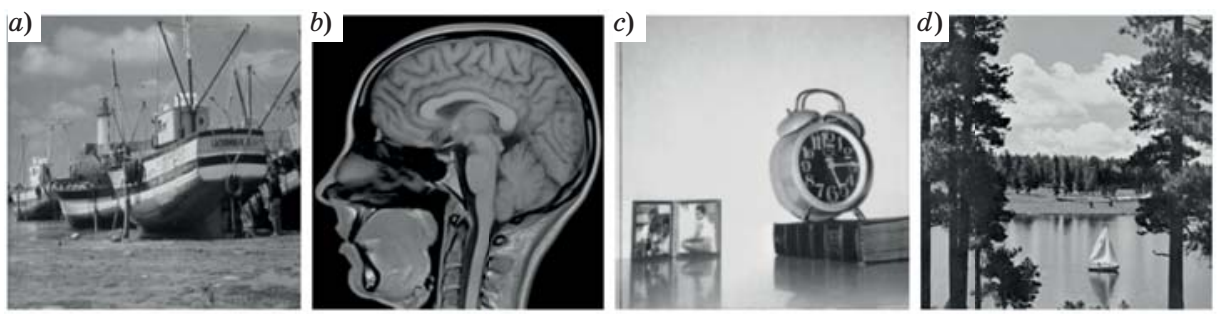

Fig. 1. Test images: $a-$ Boat; $b-\mathrm{Head} ; c-$ Clok; $d-$ Lake 
where $u, u^{*}$ are the original image, the reconstructed or noisy image accordingly; $M$ and $N$ are the number of image pixels in rows and columns.

We also use other popular measure called SSIM (structural similarity index measure). The SSIM measure compares local patterns of pixel intensities normalized for luminance and contrast, and allows us to get more consistent with human visual characteristics [28]:

$$
\operatorname{SSIM}\left(u, u^{*}\right)=\frac{\left(2 \mu_{u} \mu_{u^{*}}+c_{1}\right)\left(2 \sigma_{u, u^{*}}+c_{2}\right)}{\left(\mu_{u}^{2}+\mu_{u^{*}}^{2}+c_{1}\right)\left(\sigma_{u}^{2}+\sigma_{u^{*}}^{2}+c_{2}\right)^{*}},
$$

where $\mu_{u}, \mu_{u^{*}}$ are the means of $u, u^{*}$ respectively; $\sigma_{u}, \sigma_{u^{*}}$ - their standard deviations; $\sigma_{u, u^{*}}-$ the covariance of two images $u$ and $u^{*} ; c_{1}=\left(K_{1} L\right)^{2}$; $c_{2}=\left(K_{2} L\right)^{2}, L$ is the dynamic range of the pixel values (255 for 8-bit grayscale images), and $K_{1} \ll 1$, $K_{2} \ll 1$ are small constants.

For our experiments, we set tolerance in (19): $\sigma=0.0001$ and $N=200$. The observed images in our experiments are simulated as follows. To test different noise levels, the noisy images are generated by Poisson noise with some fixed peak $I_{\max }$, and by Gaussian noise with standard deviation $\sigma_{g}$. Empirically, all of the compared methods perform image denoising with their optimal parameters. All images are processed with the equivalent parameters $\lambda=0.4, \beta=0.6$, which gave the best restoration results. For our models, we set $\eta_{1}=5, \eta_{2}=5$ and $\eta_{3}=1$.
In Figures 2, $a-d$ and $3, a-d$ we exhibit the results of compared methods for noise levels $I_{\text {max }}=120, \sigma_{g}=5$ and $I_{\text {max }}=60, \sigma_{g}=5$.

For a better visual comparison, we show some details of the restored images in Fig. 4 for noise levels $I_{\max }=120, \sigma_{g}=5$, and in Fig. 5 for $I_{\max }=60, \sigma_{g}=5$. In these Figures, we include details of the noisy and original images. It can be seen that our method gives even better visual improvement than the other two methods. For the comparison of the performance quantitatively, the measures of PSNR and SSIM values are reported in Tables 1 and 2 . In each of the Tables, we include the PSNR and SSIM values for noisy images and recovered images, and the average results over test images for each method are shown. The better restored results are highlighted in bold.

In Figures $6, a-d$ and $7, a-d$, we also show the results details of compared methods for noise levels $I_{\max }=120, \sigma_{g}=10$ and $I_{\max }=60, \sigma_{g}=10$, respectively. We report the PSNR and SSIM values for noisy images and recovered images in Tables 3 and 4 . The average results over test images also appear in last row of each table. The better restored results are highlighted in bold.

From Figures, we can see that the images recovered by our proposed model are better quality than those of the compared approaches. Beside, the measurable comparisons reported in Tables 1-4, the our proposed approach gets higher PSNR, SSIM values than those of the TVPG and TGV approaches. It indicates the competitive performance of the proposed method for denoising image corrupted by MPGN.
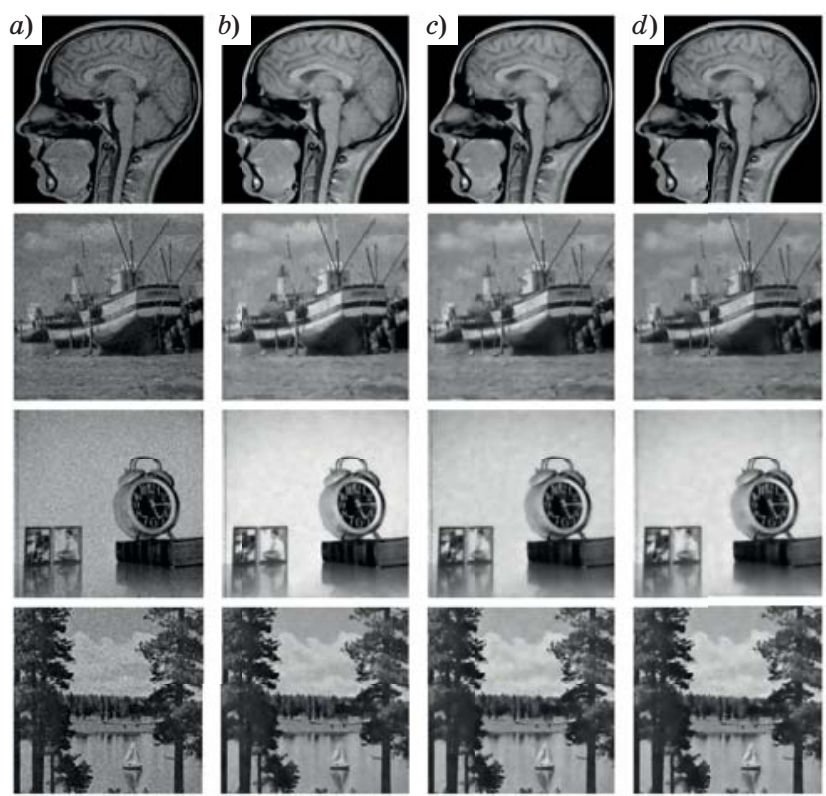

- Fig. 2. Recovered results for the test images with noise level $I_{\max }=120, \sigma_{g}=0.5: a-$ Noisy; $b-$ TVPG; $c-\mathrm{TGV} ; d-$ Ours
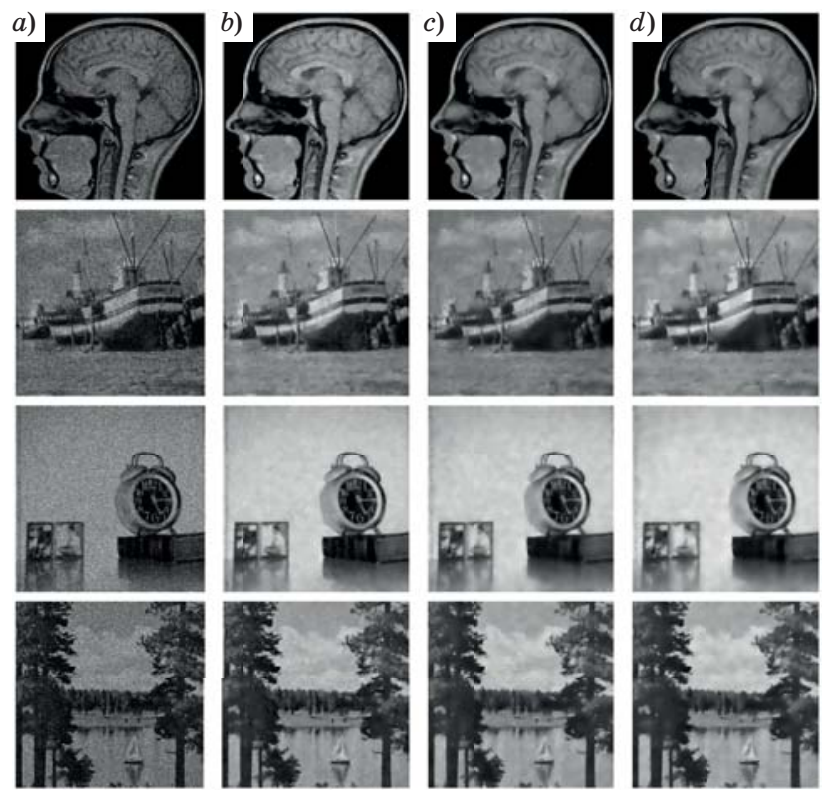

Fig. 3. Recovered results for the test images with noise level $I_{\max }=60, \sigma_{g}=0.5: a-$ Noisy; $b-$ TVPG; $c-$ $\mathrm{TGV} ; d-$ Ours 

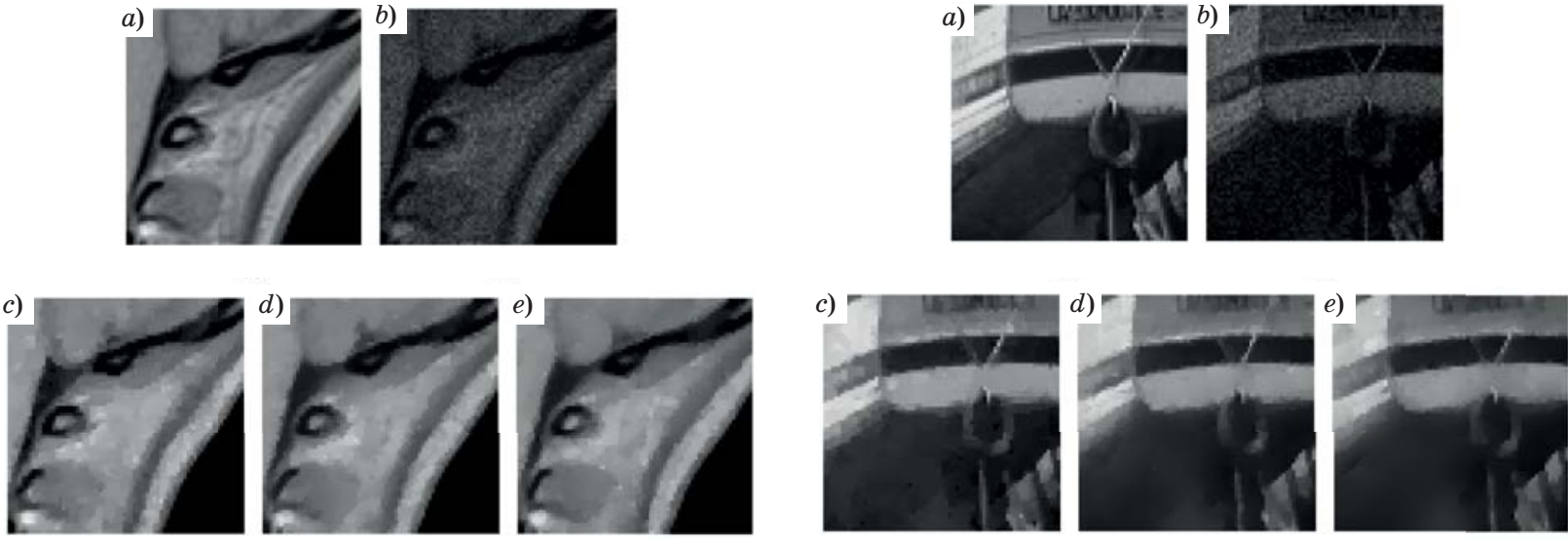

- Fig. 4. The zoom-in part of the recovered images in first row and in second row of Fig. 2: $a-$ details of original images; $b$ - details of noisy images; $c$ - details of restored images by TVPG; $d$ - details of restored images by TGV; $e-$ details of restored images by our approach
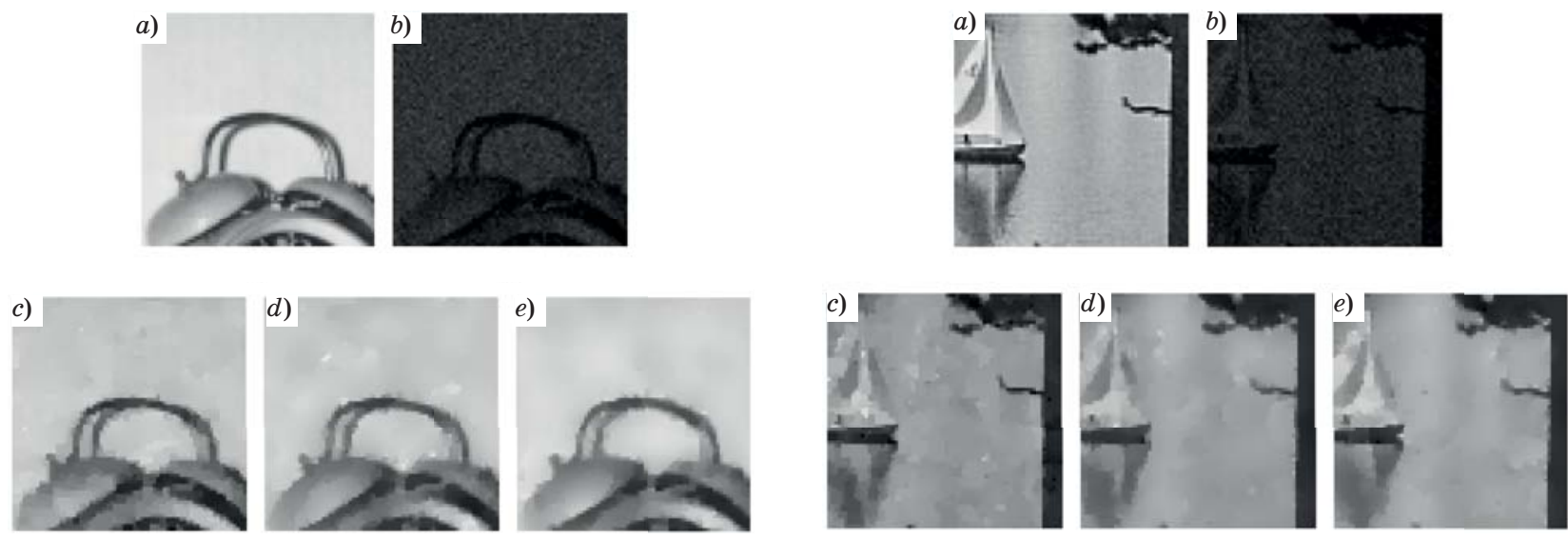

- Fig. 5. The zoom-in part of the recovered images in third row and in second row of Fig. 3: $a-$ details of original images; $b$ - details of noisy images; $c$ - details of restored images by TVPG; $d-$ details of restored images by TGV; $e-$ details of restored images by our approach

Table 1. PSNR and SSIM values for noisy images and restored images with noise level $I_{\max }=120, \sigma_{g}=5$

\begin{tabular}{|c|c|c|c|c||c|c|c|c|}
\hline \multirow{2}{*}{ Image } & \multicolumn{4}{|c||}{ PSNR } & \multicolumn{4}{c|}{ SSIM } \\
\cline { 2 - 10 } & Noisy & TGV & TVPG & Ours & Noisy & TGV & TVPG & Ours \\
\hline Board & 20.5670 & 26.9777 & 27.1435 & $\mathbf{2 7 . 5 8 2 3}$ & 0.5482 & 0.7688 & 0.7749 & $\mathbf{0 . 7 8 1 2}$ \\
\hline Clock & 15.3632 & 24.2404 & 25.9160 & $\mathbf{2 6 . 4 6 5 8}$ & 0.36742 & 0.8856 & 0.8884 & $\mathbf{0 . 8 9 5 6}$ \\
\hline Lake & 18.6823 & 24.7286 & 24.7002 & $\mathbf{2 5 . 7 1 4 1}$ & 0.61996 & 0.7649 & 0.7779 & $\mathbf{0 . 7 8 6 4}$ \\
\hline Head & 20.7322 & 26.9048 & 27.9500 & $\mathbf{2 8 . 8 8 7 4}$ & 0.60745 & 0.8624 & 0.8657 & $\mathbf{0 . 8 7 3 9}$ \\
\hline Average & 18.8362 & 25.7129 & 26.4274 & $\mathbf{2 7 . 1 6 2 4}$ & 0.5358 & 0.8204 & 0.8267 & $\mathbf{0 . 8 3 4 3}$ \\
\hline
\end{tabular}

- Table 2. PSNR and SSIM values for noisy images and restored images with noise level $I_{\max }=60, \sigma_{g}=5$

\begin{tabular}{|c|c|c|c|c||c|c|c|c|}
\hline \multirow{2}{*}{ Image } & \multicolumn{4}{|c||}{ PSNR } & \multicolumn{4}{c|}{ SSIM } \\
\cline { 2 - 10 } & Noisy & TGV & TVPG & Ours & Noisy & TGV & TVPG & Ours \\
\hline Board & 18.6799 & 24.0460 & 24.7064 & $\mathbf{2 5 . 1 7 1 3}$ & 0.3871 & 0.6701 & $\mathbf{0 . 6 8 1 8}$ & $\mathbf{0 . 6 9 3 1}$ \\
\hline Clock & 13.0537 & 24.3635 & 24.4234 & $\mathbf{2 5 . 5 3 4 5}$ & 0.2600 & 0.8409 & 0.8423 & $\mathbf{0 . 8 5 8 7}$ \\
\hline Lake & 16.339 & 22.0954 & 22.4670 & $\mathbf{2 2 . 8 3 7 9}$ & 0.4735 & 0.6762 & 0.6877 & $\mathbf{0 . 6 9 2 0}$ \\
\hline Head & 16.7107 & 25.2752 & 25.6161 & $\mathbf{2 6 . 4 4 1 1}$ & 0.5736 & 0.7724 & 0.7923 & $\mathbf{0 . 8 0 8 7}$ \\
\hline Average & 16.1958 & 23.9450 & 24.3032 & $\mathbf{2 4 . 9 9 6 2}$ & 0.4235 & 0.7399 & 0.7510 & $\mathbf{0 . 7 6 3 1}$ \\
\hline
\end{tabular}



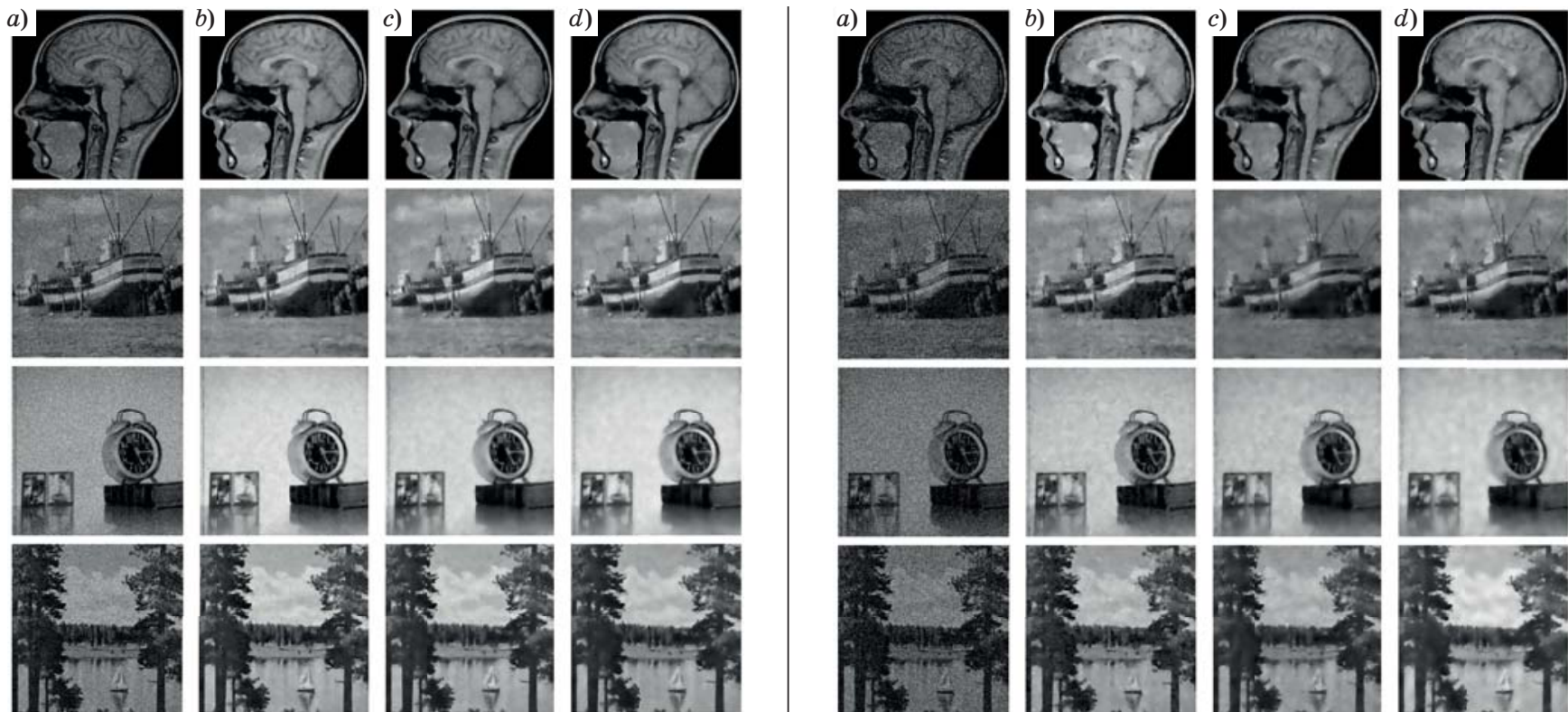

- Fig. 6. Recovered results for the test images with noise level $I_{\max }=120, \sigma_{g}=10: a-$ Noisy; $b-$ TVPG;

- Fig. 7. Recovered results for the test images with noise level $I_{\max }=60, \sigma_{g}=10: a-$ Noisy; $b-$ TVPG; $c-$ $c-\mathrm{TGV} ; d-$ Ours TGV; $d-$ Ours

- Table 3. PSNR and SSIM values for noisy images and restored images with noise level $I_{\max }=120, \sigma_{g}=10$

\begin{tabular}{|c|c|c|c|c||c|c|c|c|}
\hline \multirow{2}{*}{ Image } & \multicolumn{4}{|c||}{ PSNR } & \multicolumn{4}{c|}{ SSIM } \\
\cline { 2 - 11 } & Noisy & TGV & TVPG & Ours & Noisy & TGV & TVPG & Ours \\
\hline Board & 19.7547 & 24.7675 & 25.9887 & $\mathbf{2 6 . 1 7 3 3}$ & 0.4376 & $\mathbf{0 . 7 2 5 5}$ & 0.7218 & $\mathbf{0 . 7 3 1 6}$ \\
\hline Clock & 14.5413 & 22.4326 & 24.6121 & $\mathbf{2 5 . 9 6 8 7}$ & 0.2980 & 0.8571 & 0.8421 & $\mathbf{0 . 8 7 4 9}$ \\
\hline Lake & 17.805 & 23.3247 & 23.7328 & $\mathbf{2 4 . 2 7 8 7}$ & 0.5191 & 0.7249 & 0.7270 & $\mathbf{0 . 7 3 9 6}$ \\
\hline Head & 16.031 & 26.3812 & 26.5097 & $\mathbf{2 7 . 0 1 1 9}$ & 0.6075 & 0.8154 & 0.8292 & $\mathbf{0 . 8 3 5 8}$ \\
\hline Average & 17.0330 & 24.2265 & 25.2108 & $\mathbf{2 5 . 8 5 8 2}$ & 0.4655 & 0.78073 & $\mathbf{0 . 7 8 0 0}$ & $\mathbf{0 . 7 9 5 5}$ \\
\hline
\end{tabular}

- Table 4. PSNR and SSIM values for noisy images and restored images with noise level $I_{\max }=60, \sigma_{g}=10$

\begin{tabular}{|c|c|c|c|c||c|c|c|c|}
\hline \multirow{2}{*}{ Image } & \multicolumn{4}{|c||}{ PSNR } & \multicolumn{4}{c|}{ SSIM } \\
\cline { 2 - 10 } & Noisy & TGV & TVPG & Ours & Noisy & TGV & TVPG & Ours \\
\hline Board & 17.5737 & 23.3837 & 23.5885 & $\mathbf{2 3 . 8 1 8 9}$ & 0.2566 & 0.6060 & $\mathbf{0 . 6 0 5 4}$ & $\mathbf{0 . 6 2 1 5}$ \\
\hline Clock & 12.2833 & 24.3595 & 24.2930 & $\mathbf{2 4 . 4 3 2 0}$ & 0.1793 & 0.7965 & 0.7726 & $\mathbf{0 . 8 1 5 0}$ \\
\hline Lake & 14.6131 & 20.8641 & 20.8629 & $\mathbf{2 1 . 5 5 2 3}$ & 0.3230 & 0.6018 & $\mathbf{0 . 6 0 9 7}$ & $\mathbf{0 . 6 2 0 7}$ \\
\hline Head & 14.1531 & 23.4717 & 24.2758 & $\mathbf{2 4 . 6 9 0 4}$ & 0.4588 & 0.7304 & $\mathbf{0 . 7 3 8 6}$ & $\mathbf{0 . 7 4 9 6}$ \\
\hline Average & 14.6558 & 23.0198 & 23.2551 & $\mathbf{2 3 . 6 2 3 4}$ & 0.3044 & 0.6837 & $\mathbf{0 . 6 8 1 6}$ & $\mathbf{0 . 7 0 1 7}$ \\
\hline
\end{tabular}

\section{Conclusions}

In this paper, we have investigated a second-order $T G V_{\alpha}^{2}$ based model for denoising image corrupted by MPGN. Computationally, an alternating minimization algorithm is employed for solving the proposed optimization problem. Finally, compared with several existing state-of-the-art approaches, the experiments demonstrate competitive performance of the proposed method. 


\section{Financial support}

This work was supported by The University of Danang, University of Science and Technology, code number of Project T2020-02-33.

\section{Appendix}

Definition 1 [20, 23-25]. Let $\Omega \subset \mathbb{R}^{2}$ be a bound domain, $k>1$ and $\alpha=\left(\alpha_{0}, \alpha_{1}\right)>0$.

Then the total generalized variation of order $k$ with weight $\alpha$ for $u \in L^{1}(\Omega)$ is defined as the value of the functional:

$$
\begin{gathered}
T G V_{\alpha}^{2}(u)=\sup \left\{\int_{\Omega} u \operatorname{div}^{2} \vartheta \mathrm{d} x \mid \vartheta \in C_{C}^{2}\left(\Omega, \mathbb{S}^{d \times d}\right),\right. \\
\left.\|\vartheta\|_{\infty} \leq \alpha_{0},\|\operatorname{div} \vartheta\|_{\infty} \leq \alpha_{1}\right\},
\end{gathered}
$$

where $d$ denotes the dimension of images, $C_{C}^{2}\left(\Omega, \mathbb{S}^{d \times d}\right)$ is the space of compactly supported symmetric $d \times d$ matrix fields, $\mathbb{S}^{d \times d}$ is the set of all symmetric $d \times d$ matrices,

$$
(\operatorname{div} \vartheta)_{i}=\sum_{j=1}^{d} \frac{\partial \vartheta_{i j}}{\partial x_{j}},\left(\operatorname{div}^{2} \vartheta\right)_{i}=\sum_{i=1, j=1}^{d} \frac{\partial^{2} \vartheta_{i j}}{\partial x_{i} \partial x_{j}} .
$$

The infinite norms of $\theta$ and $\operatorname{div} \theta$ are given by

$$
\|\vartheta\|_{\infty}=\sup _{x \in \Omega}\left(\sum_{i=1, j=1}^{d}\left|\vartheta_{i j}\right|^{2}\right)^{\frac{1}{2}} ;
$$

\section{References}

1. Pham Cong Thang, Andrei V. Kopylov. Tree-serial parametric dynamic programming with flexible prior model for image denoising. Computer Optics, 2018, vol. 42(5), pp. 838-845.

2. Le T., Chartrand R., Asaki T. J. A variational approach to reconstructing images corrupted by Poisson noise. Journal of Mathematical Imaging and Vision, 2007, vol. 27, pp. 257-263.

3. Li J., Shen Z., Yin R., Zhang X. A reweighted method for image restoration with Poisson and mixed Poisson-Gaussian noise. Inverse Problems \& Imaging, 2015, vol. 9 (3), pp. 875-894.

4. Chouzenoux E., Jezierska A., Pesquet J. C., Talbot H. A convex approach for image restoration with exact Poisson-Gaussian likelihood. SIAM Journal on Imaging Sciences, 2015, vol. 8(4), pp. 2662-2682.

5. Benvenuto F., Camera A. L., Theys C., Ferrari A., Lanteri H., Bertero M. The study of an iterative method for the reconstruction of images corrupted by Poisson and Gaussian noise. Inverse Problems, 2008, vol. 24(3), pp. 35016.

$$
\|\operatorname{div} \vartheta\|_{\infty}=\sup _{x \in \Omega}\left(\sum_{j=1}^{d}\left|(\operatorname{div} \vartheta)_{j}(x)\right|^{2}\right)^{\frac{1}{2}}
$$

Definition 2 [20, 23-25]. The space of functions of bounded generalized variation ( $B G V)$ is defined as follows:

$$
\begin{gathered}
B G V^{2}(\Omega)=\left\{u \in L^{1}(\Omega) \mid T G V_{\alpha}^{2}(u)<\infty\right\}, \\
\|u\|_{B G V^{2}}=\|u\|_{1}+T G V_{\alpha}^{2}(u) .
\end{gathered}
$$

$B G V^{2}(\Omega)$ is a Banach space independent of the weight vector $\alpha, T G V_{\alpha}^{2}$ is a seminorm and a convex function in $B G V^{2}(\Omega)$. Subsequently, we denote the spaces $U=C_{C}^{2}(\Omega, \mathbb{R}), V=C_{C}^{2}\left(\Omega, \mathbb{R}^{2}\right)$ and $G=C_{C}^{2}\left(\Omega, \mathbb{S}^{2 \times 2}\right)$.

\section{Proof for Theorem 1.}

Let $u^{(k)}$ be a bounded minimizing sequence. By the compactness property in the space of bound variation $B V(\Omega)$, there exists $u^{*} \in B V(\Omega)$, such that $u^{(k)}$ converges weakly to $u^{*} \in B V(\Omega)$ and $u^{(k)}$ converges strongly to $u^{*}$ in $L^{1}(\Omega)$. According to [7, 23-26], we know that the functions $T G V_{\alpha}^{2}(u)$ and data fidelity term are all lower semi-continuous, proper and convex; and according to Fatou's lemma [29], we have

$$
E(u) \geq E\left(u^{*}\right)
$$

Thus, $u^{*}$ is a minimizer of the optimization problem (4).

6. Lanza A., Morigi S., Sgallari F., Wen Y. W. Image restoration with Poisson-Gaussian mixed noise. Computer Methods in Biomechanics and Biomedical Engineering: Imaging and Visualization, 2014, vol. 2(1), pp. 12-24.

7. Calatroni L., De Los Reyes J., and Schronlieb C. Infimal convolution of data discrepancies for mixed noise removal. SIAM Journal on Imaging Sciences, 2017, vol. 10(3), pp. 1196-1233.

8. Pham C. T., Gamard G., Kopylov A., Tran T. T. T. An algorithm for image restoration with mixed noise using total variation regularization. Turkish Journal of Electrical Engineering and Computer Sciences, 2018, vol. 26(6), pp. 2831-2845.

9. Chambolle A. An algorithm for total variation minimization and applications. Journal of Mathematical Imaging and Vision, 2004, vol. 20, pp. 89-97.

10. He C., Hu C., Zhang W., Shi B. A fast adaptive parameter estimation for total variation image restoration. IEEE Transactions on Image Processing, 2014, vol. 23(12), pp. 4954-4967.

11. Huang Y. M., Ng M. K., Wen Y. W. A fast total variation minimization method for image restoration. 
Multiscale Modeling and Simulation, 2008, vol. 7(2), pp. 774-795.

12. Wang Y., Yang J., Yin W., Zhang Y. A New alternating minimization algorithm for total variation image reconstruction. SIAM Journal on Imaging Sciences, 2008, vol. 1 (3), pp. 248-272.

13. Goldstein T., Osher S. The split Bregman method for L1-regularized problems. SIAM Journal on Imaging Sciences, 2009, vol. 2(2), pp. 89-97.

14. Chen H., Wang C., Song Y., and Li Z. Split bregmanized anisotropic total variationmodel for image deblurring. Journal of Visual Communication and Image Representation, 2015, vol. 31, pp. 282-293.

15. Kayyar S. H., Jidesh P. Non-local total variation regularization approach for image restoration under a Poisson degradation. Journal of Modern Optics, 2018, vol. 65, pp. 2231-2242.

16. Lysaker M., Lundervold A., Tai X.-C. Noise removal using fourth order partial differential equation with applications to medical magnetic resonance images in space and time. IEEE Transactions on Image Process ing, 2003, vol. 12 , pp. 1579-1590.

17. Zhang J., Chen R., Deng C., and Wang S. Fast linearized augmented Lagrangian method for Euler's elastica model. Numerical Mathematics: Theory, Methods and Applications, 2017, vol. 10, pp. 98115.

18.Zhu W., Tai X. C., Chan T. A fast algorithm for a mean curvature based image denoising model using augmented Lagrangian method. In: Efficient Algorithms for Global Optimization Methods in Computer Vision. Lecture Notes in Computer Science. Springer, Berlin, Heidelberg, 2014. Vol. 8293. Pp. 104118.

19. Myllykoski M., Glowinski R., Karkkainen T., Rossi T. A new augmented Lagrangian approach for -mean curvature image denoising. SIAM Journal on Imaging Sciences, 2015, vol. 8(1), pp. 95-125.
20. Bredies K., Kunisch K., Pock T. Total generalized variation. SIAM Journal on Imaging Sciences, 2010, vol. 3(3), pp. 492-526.

21. Bredies L. K, Dong Y., Hintermller M. Spatially dependent regularization parameter selection in total generalized variation models for image restoration. International Journal of Computer Mathematics, 2013, vol. 90(1), pp. 109-123.

22. He C., Hu C., Yang X., He H., Zhang Qi. An adaptive total generalized variation model with augmented Lagrangian method for image denoising. Mathematical Problems in Engineering, 2014, vol. 2014, Article ID 157893, $11 \mathrm{p}$.

23. Knoll F., Bredies K., Pock T., Stollberger R. Second order total generalized variation (TGV) for MRI. Magnetic Resonance in Medicine, 2011, vol. 65(2), pp. 480-491.

24. Guo W., Qin J., Yin W. A new detail-preserving regularity scheme. SIAM Journal on Imaging Sciences, 2014, vol. 7(2), pp. 1309-1334.

25. Liu X. Augmented Lagrangian method for total generalized variation based Poissonian image restoration. Computers \& Mathematics with Applications, 2016, vol. 71(8), pp. 1694-1705.

26. Pham C. T., Tran T. T. T., Gamard G. An efficient total variation minimization method for image restoration. Informatica, 2020, vol. 31(3), pp. 539-560.

27. Micchelli C. A., Shen L., Xu Y. Proximity algorithms for image models: denoising. Inverse Problem, 2011, vol. 27(4), 045009.

28. Wang Z., Bovik A. C., Sheikh H. R., Simoncelli E. P. Image quality assessment: From error visibility to structural similarity. IEEE Transactions on Image Processing, 2004, vol. 13, pp. 600-612.

29. Feinberg E. A., Kasyanov P. O., Zadoianchuk N. V. Fatou's lemma for weakly converging probabilities. Theory of Probability \& its Applications, 2014, vol. 58(4), pp. 683-689.

УДК 004.93

doi:10.31799/1684-8853-2021-2-20-32

Модель на основе полной обобщенной вариации второго порядка для восстановления изображений со смешанным пуассоновско-гауссовским шумом

Фам Конг Тханга, PhD, преподаватель, orcid.org/0000-0002-6428-102X, pcthang@dut.udn.vn

Чан Тхи Тху Тхао ${ }^{\sigma}$ магистр, преподаватель, orcid.org/0000-0001-7705-2405

Нгуен Тхань Конга, магистр, специалист, orcid.org/0000-0002-8060-0238

Во Дык Хоанга, $\mathrm{PhD}$, преподаватель, orcid.org/0000-0002-6974-9023

ауниверситет науки и техники, Нгуэн Лунг Банг, 54, Дананг, 550000, Вьетнам

бУниверситет экономики, Нгу Ханх Сон, 71, Дананг, 550000, Вьетнам

Введение: восстановление изображений играет важную роль в обработке цифровых изображений. Распространенной проблемой восстановления изображений является шумоподавление. В области шумоподавления изображений существует множество моделей шума, одной из них можно назвать модель смешанного пуассоновско-гауссовского шума, которая с недавнего времени вызывает большой интерес. Цель: разработка модели шумоподавления изображений, искаженных смешанным пуассоновско-гауссовским шумом, и алгоритма для решения результирующей задачи минимизации. Результаты: предложена новая модель полной вариации для восстановления изображения со смешанным пуассоновско-гауссовским шумом на основе полной обобщенной 
вариации второго порядка. Для решения рассматриваемой задачи оптимизации применяется эффективный алгоритм чередующейся минимизации. В качестве иллюстрации, в сравнение с родственными методами, представлены экспериментальные результаты, свидетельствующие о высокой эффективности предлагаемого подхода. Практическая значимость: разработанная модель позволяет удалить смешанныый пуассоновско-гауссовский шум на цифровых изображениях с сохранением границ. Приведенные численные результаты демонстрируют конкурентоспособные характеристики предложенной модели для шумоподавления изображений, искаженных смешанным пуассоновско-гауссовским шумом.

Ключевые слова - шумоподавление изображения, полная вариация, минимизация, смешанный пуассоновско-гауссовский шум.

Для цитирования: Pham C. T., Tran T. T. T., Nguyen T. С., Vo D. H. Second-order total generalized variation based model for restoring images with mixed Poisson - Gaussian noise. Инфорлационно-управляющие системы, 2021, № 2, с. 20-32. doi:10.31799/16848853-2021-2-20-32

For citation: Pham C. T., Tran T. T. T., Nguyen T. C., Vo D. H. Second-order total generalized variation based model for restoring images with mixed Poisson - Gaussian noise. Informatsionno-upravliaiushchie sistemy [Information and Control Systems], 2021, no. 2, pp. 20-32. doi:10.31799/1684-8853-2021-2-20-32

\section{ПАМЯТКА АМЯ АВТОРОВ}

Поступающие в редакиию статьи проходят обязательное рецензирование.

При наличии положительной рецензии статья рассматривается редакционной коллегией. Принятая в печать статья направляется автору для согласования редакторских правок. После согласования автор представляет в редакцию окончательный вариант текста статьи.

Процедуры согласования текста статьи могут осуществляться как непосредственно в редакции, так и по e-mail (ius.spb@gmail.com).

При отклонении статьи редакция представляет автору мотивированное заключение и рецензию, при необходимости доработать статью - рецензию.

Редакиия журнала наполинает, что ответственность за достоверность и точность реклалных материалов несут реклалодатели. 